\title{
Dinâmica do Emprego e Custos de Ajustamento na Indústria do Rio Grande do Sul ${ }^{\star}$
}

\author{
- PAUlo de ANDRAde Jacinto*
}

- Eduardo Pontual Ribeiro**

\begin{abstract}
RESUMO
O presente estudo tem como propósito fazer uma análise empírica da estrutura dos custos de ajustamento do emprego industrial usando dados do Brasil. As estruturas teóricas para custos de ajustamento do emprego encontradas na literatura podem ser agrupadas em custos convexos (que incluem a popular função quadrática) e não-convexos (como custos fixos). Seguindo o trabalho de Varejão e Portugal (2007), empregamos metodologias complementares para a identificação da estrutura de custos de ajustamento de modo inovador no Brasil. Primeiro, usando matrizes de transição entre regimes de emprego. Segundo, empregando modelos de sobrevivência, que são mais gerais que os modelos de matriz de transição Markoviana. Os resultados obtidos sugerem que os custos de ajustamento não-convexos têm mais apoio nos dados do que o modelo usual de custos de ajustamentos quadráticos.
\end{abstract}

\section{Palavras-Chave}

custos de ajustamento, modelos de duração, demanda por trabalho, emprego, microdados

\begin{abstract}
We evaluate the structure of adjustment costs in manufacturing employment using Brazilian firm data. While quadratic adjustment costs are widely used this type of costs are not compatible with the extensive within sector gross job flows. Following Varejão and Portugal (2007), we use survival analysis as an empirical tool, as previous results associate non-convex adjustment costs with inactivity time dependent exit probabilities. Our estimates using quarterly firm level data from manufacturing industries, from 199I to 2004 soundly reject time independent exit probabilities from labor adjustment inactivity, a result that is compatible with non-convex adjustment costs.
\end{abstract}

\section{KEYWORDS}

adjustment costs, labor demand, duration models

\section{JEL CLASSIFICATION}

J23, J63

\footnotetext{
- Este trabalho é uma versão revisada de partes da tese de doutorado do primeiro autor no PPGE/UFRGS. Eduardo Ribeiro recebeu apoio do CNPq para esta pesquisa. Agradecemos à FIERGS pelo acesso aos dados e aos comentários de Giácomo Balbinotto, Naércio Menezes, Luiz G. Scorzafave. Erros são de nossa responsabilidade.

* Professor da FACE e do Programa de Pós-Graduação em Economia da PUCRS. Endereço para contato: Avenida Ipiranga, 668I Prédio 50, sala I00I - Porto Alegre - RS. Tel. (5I)3320-3688 Ramal 7799. E-mail: pajjap@hotmail. com.

** Instituto de Economia - Universidade Federal do Rio de Janeiro. Endereço para contato: Av. Pasteur, 250, Térreo - Urca - Rio de Janeiro - RJ. Tel: (2I)3873-5246. E-mail: eribeiro@ie.ufrj.br.

(Recebido em agosto de 2007. Aceito para publicação em setembro de 2008).
} 


\section{$1 \quad$ INTRODUÇÃO}

Nos anos 90, consolidou-se o fato estilizado de que as variações no emprego nas empresas eram substancialmente heterogêneas e apresentavam uma dinâmica diferente do comportamento do emprego agregado (DAVIS; HALTIWANGER, 1999). No Brasil, foi identificado por Corseuil e Servo (2007) que aproximadamente um terço do emprego na economia em cada ano estava crescendo ou diminuindo nas empresas, embora a variação líquida agregada fosse menos de cinco pontos percentuais. Segundo Hamermesh e Pfann (1996a), estes fatos têm implicações na compreensão dos efeitos de mudanças nos determinantes do emprego.

Para entender os efeitos de mudanças estruturais, tecnológicas e conjunturais no emprego industrial o procedimento mais utilizado é a estimação de uma função de demanda por trabalho. A partir da forma funcional e estimativa dos parâmetros dessa função é possível obter contrafactuais para análise de políticas econômicas sobre produção e custo do trabalho. Todavia, esse procedimento exige a especificação apropriada para a estrutura de custos de ajustamento do emprego no tempo, que não parece compatível com a heterogeneidade intrassetorial encontrada. Vale mencionar que a forma dos custos de ajustamento influencia as elasticidades produto e salário do emprego observadas.

Por custos de ajustamento entendem-se aqueles custos diretos e indiretos gerados por alterações na quantidade da mão de obra empregada na firma. De modo geral, os custos de contratação incorporam os custos da firma em anunciar as vagas disponíveis, entrevistar e selecionar os candidatos e treinar os novos trabalhadores; por sua vez, os custos de demissão normalmente estão previstos por lei, como o aviso prévio e demais indenizações. Em custos indiretos inclui-se o custo de oportunidade em termos de produção perdida enquanto o novo trabalhador não adquire a mesma produtividade do trabalhador que foi substituído.

A representação analítica destes custos é feita por meio de uma função de custos de ajustamento. A mais usual possui a forma quadrática e simétrica, embora estudos mais recentes tenham empregado uma forma quadrática e assimétrica. A primeira admite que os custos na contratação sejam semelhantes aos de demissão, ao passo que a segunda permite que esses custos sejam diferentes. Funções quadráticas geram um padrão de ajuste da mão de obra contínuo, suave e sem movimentos bruscos a partir de uma sequência de choques de rentabilidade percebidos pela empresa (HAMERMESH, 1993). Dessa forma, a estratégia ótima da empresa, independentemente ou não de simetria, é ajustar o número de trabalhadores de forma gradual e sem interrupções. 
A partir do estudo de Hamermesh (1989), funções de custos de ajustamento alternativas e mais complexas incorporando custos fixos (lumpy costs) ou lineares (piecewise costs) passaram a ser consideradas de modo mais significativo apesar de já terem sido propostas em 1962 por Walter Oi. A principal característica dessas funções encontrase na descontinuidade dos ajustes no emprego, o que implica uma ausência de ajuste gradual na mão de obra e a existência de períodos de inatividade frente a uma sequência de choques. Com isso a elasticidade-emprego observada passa a depender do tamanho dos choques e pode apresentar descontinuidades.

Estudos como o de Caballero, Engel e Haltiwanger (1997), Cooper e Willis (2001) e Varejão e Portugal (2007), levando em consideração a heterogeneidade nas variações no emprego observadas por Davis e Haltiwanger (1999) e Hamermesh (1989), ao invés de focar a forma funcional detalhada dos custos de ajustamento (quadráticos e simétricos, quadráticos e assimétricos, custos fixos ou lineares), focalizam suas análises na verificação do tipo geral de custos de ajustamento, ou seja, convexos ou não-convexos. A convexidade é associada a ajustes frequentes, porém, com variações no emprego diluídas ao longo do tempo, ao passo que os custos de ajustamentos não-convexos geram ajustamentos pouco frequentes e com movimentos bruscos na mão de obra empregada, caracterizando períodos de inatividade e ausência de ajuste. É uma maneira mais geral de abordar os custos de ajustamento, uma vez que se busca conhecer a estrutura de custos sem impor maiores restrições sobre as funções de custos de ajustamento das firmas. O interesse está em identificar o tipo geral com o mínimo de hipóteses identificadoras sobre a realidade.

$\mathrm{Na}$ literatura econômica, essa questão tem sido tratada através da estimação de modelos estruturais de demanda por trabalho que incorporam estruturas alternativas de custos de ajustamento. Os resultados mostram que modelos de demanda por trabalho não-linear, que correspondem à estrutura de custos de ajustamento não-convexa, ajustam-se melhor aos dados de empresas. Destacam-se os trabalhos de Hamermesh (1989, 1990 e 1992), que examinaram dados mensais de firmas individuais no nível do estabelecimento, descobrindo que a série agregada aparentava um ajustamento suave, sem movimentos bruscos, ao passo que no nível individual da firma o ajustamento do emprego era extremamente fixo.

A literatura de investimentos também trata da questão dos custos de ajustamento do estoque de capital. Nielsen e Schiantarelli (2003) e Cooper et al. (1999) mostram que o tipo de custo de ajustamento pode ser identificado através de métodos de análise de sobrevivência, ao invés da estimação de modelos estruturais de demanda por investimento. Estes autores concluem que para empresas o modelo de custos de ajustamento não-convexos possui maior apoio empírico do que custos convexos. 
No Brasil, os estudos de Estevão (1991), Pereira e Gonzaga (1998), Meyer (1998), Ambrózio (1999), Magnusson (2000) e Gonzaga e Corseuil (2001) empregam custos de ajustamento quadráticos que consideram funções de demanda por emprego do tipo de ajustamento parcial, aplicados a dados agregados (setoriais). O tipo de custo de ajustamento é suposto sem uma avaliação da validade desta suposição. Caballero et al. (1997) mostram que com o uso de dados setoriais (agregados) a estrutura de custos de ajustamento tende a parecer quadrática (convexa), embora estes custos nas empresas (microdados) possam ser bem diferentes numa aplicação moderna da famosa falácia da composição. ${ }^{1}$ Assim, o presente estudo tem como propósito fazer uma análise empírica da estrutura dos custos de ajustamento do emprego em indústrias brasileiras a partir de dados microeconômicos para identificar o tipo de custos nas empresas. Os dados representam empresas industriais do Rio Grande do Sul entre os anos de 1991 e 2004.

Para tanto, além dessa breve introdução, o trabalho está organizado em quatro seções. Na segunda, a base de dados utilizada é descrita. Na terceira, as matrizes de transição entre os regimes de emprego são apresentadas. Na quarta, a análise estatística da duração do ajustamento do emprego é utilizada para diferenciar as estruturas de custos de ajustamento do emprego. Por fim, a seção cinco apresenta as considerações finais. Adiantando as conclusões, os resultados mostram que os custos de ajustamento não-convexos têm apoio nos dados, e que o modelo dinâmico com custos de ajustamento quadrático, muito explorado nos estudos empíricos no Brasil, não parece ser o modelo mais adequado.

\section{AS EMPRESAS ESTUDADAS E AS HIPÓTESES DE IDENTIFICAÇÃO}

Os dados utilizados para identificar a estrutura de custos de ajustamento e analisar a dinâmica da demanda por trabalho na indústria foram obtidos junto à Federação das Indústrias do Estado do Rio Grande do Sul (FIERGS) e têm origem na Pesquisa Indicadores Industriais da Confederação Nacional das Indústrias (CNI). O âmbito da pesquisa inclui as empresas que fazem parte do cadastro próprio da FIERGS, e as unidades de informação são as empresas industriais e suas filiais localizadas no Estado do Rio Grande do Sul. A classificação de atividades da pesquisa é a mesma do cadastro da Relação Anual de Informações Sociais do ano de 1988 (RAIS88), ou seja, corresponde à classificação utilizada pela Classificação Nacional de Atividades Econômicas do ano de 1973 (CNAE-73), tanto para o gênero industrial

1 A falácia da composição ocorre quando alguém infere que algo é verdadeiro no todo a partir do fato de que ela é verdadeira apenas em parte do todo. Este argumento pode ser usado (de modo reverso) para admitir que os custos de ajustamento são convexos para empresas individuais a partir da estimação de modelos com dados agregados. 
quanto para o subgênero. ${ }^{2}$ A amostra engloba o período de janeiro de 1991 a maio de 2004.

A amostra utilizada pela FIERGS apresenta um viés para médias e grandes empresas e inclui apenas empresas formais. O processo de amostragem utilizado na formação do painel de empresas ${ }^{3}$ informantes é incidental ${ }^{4}$, tendo como um dos critérios empregados na formação desse painel a seleção de unidades informantes do cadastro da FIERGS responsáveis por $50 \%$ do número de empregados do gênero. ${ }^{5}$ No caso particular daqueles gêneros não explicitados individualmente, ou seja, na classe de "outros gêneros", o percentual passa a ser igual a $20 \%$ do número de empregados. Uma apresentação detalhada sobre a distribuição das empresas por tamanho, número de empresas, valores médios das vendas reais e custo médio do trabalho por quinquênio encontra-se nos Anexos. ${ }^{6}$ A estrutura amostral da pesquisa visa acompanhar tendências de curto prazo, ao invés de gerar estimativas de totais para a indústria.

Por se tratar de uma amostra incidental, uma comparação entre as séries de emprego, oriunda da Pesquisa Industrial Mensal (PIM) realizada pelo IBGE, com a série de emprego utilizada nesse trabalho, pode indicar, de modo informal, se podemos considerar a amostra utilizada pela Pesquisa Indicadores Industriais da Confederação Nacional das Indústrias (PCNI) representativa. Nesse sentido, características como a presença de uma tendência declinante do emprego ao longo do tempo no setor industrial na série de emprego gerada pela PIM devem estar presentes na série de emprego obtida pela CNI. Complementando a análise gráfica, estatisticamente, uma análise da correlação entre as duas séries também pode ser útil para mostrar se existe uma forte relação entre as duas variáveis e confirmar a representatividade ou não da amostra.

Como pode ser observado na Figura 1, a série de emprego oriunda da pesquisa da CNI para o Rio Grande do Sul (PCNI), no período 1991-2001, apresenta o mesmo comportamento observado na série do emprego da PIM para a Região Sul (PIM),

2 As unidades estão agrupadas em 16 setores ou gêneros industriais: metalúrgica, mecânica, material elétrico e de comunicações, material de transporte, madeira, mobiliário, borracha, couros e peles, química, têxtil, vestuário, calçados e artefatos de tecidos, produtos alimentares, bebidas, editorial e gráfica, fumo e diversas.

3 Sem acompanhamento demográfico de entradas e saídas e estatístico de plano amostral.

4 Se localizada no Estado, a matriz, responde às informações referentes a ela e às suas filiais no Estado. No caso das empresas, cujas matrizes localizam-se fora do Estado, as informações coletadas referem-se exclusivamente a elas, ou seja, as filiais.

5 Os gêneros incluídos na pesquisa são responsáveis por, no mínimo, $70 \%$ do valor da produção da indústria de transformação do Estado, encontrado no Censo IBGE 1985 para o início da amostra ou, para os anos mais recentes, na RAIS. Os gêneros restantes compõem a classe "Outros", utilizada na obtenção do resultado agregado para a indústria de transformação na pesquisa.

6 As informações sobre pessoal referem-se ao pessoal ocupado com vínculo empregatício no último dia de cada mês, ou seja, as movimentações de trabalhadores entre essas datas ocorridas nas empresas não são consideradas. 
ou seja, ambas as séries mostram uma trajetória de declínio do emprego industrial nos anos 90. A marcante recuperação em 1999, na pesquisa para o Rio Grande do Sul, reflete a importância do setor de calçados para o emprego no Estado, que não é tão representativo no total da Região Sul. A correlação entre as duas séries é de 0,92 , o que mostra uma forte relação entre as duas séries. Com base na análise gráfica e no índice de correlação é possível inferir que a amostra utilizada pela Pesquisa Indicadores Industriais da Confederação Nacional das Indústrias é representativa, podendo ser utilizada para estudar o comportamento do emprego no Rio Grande do Sul.

Uma primeira aproximação para conhecer o padrão de ajustamento do emprego pode ser observada na Figura 2, na qual é apresentada a distribuição das taxas de crescimento do emprego por trimestre para as empresas que aparecem na amostra por pelo menos dois trimestres contíguos. ${ }^{7}$ De modo geral, observa-se imediatamente uma concentração das taxas em zero, gerando um pico e revelando uma considerável rigidez nas alterações no emprego.

\section{FIGURA 1 - PESSOAL OCUPADO NA INDÚSTRIA (DADOS DESSAZONA- LIZADOS)}

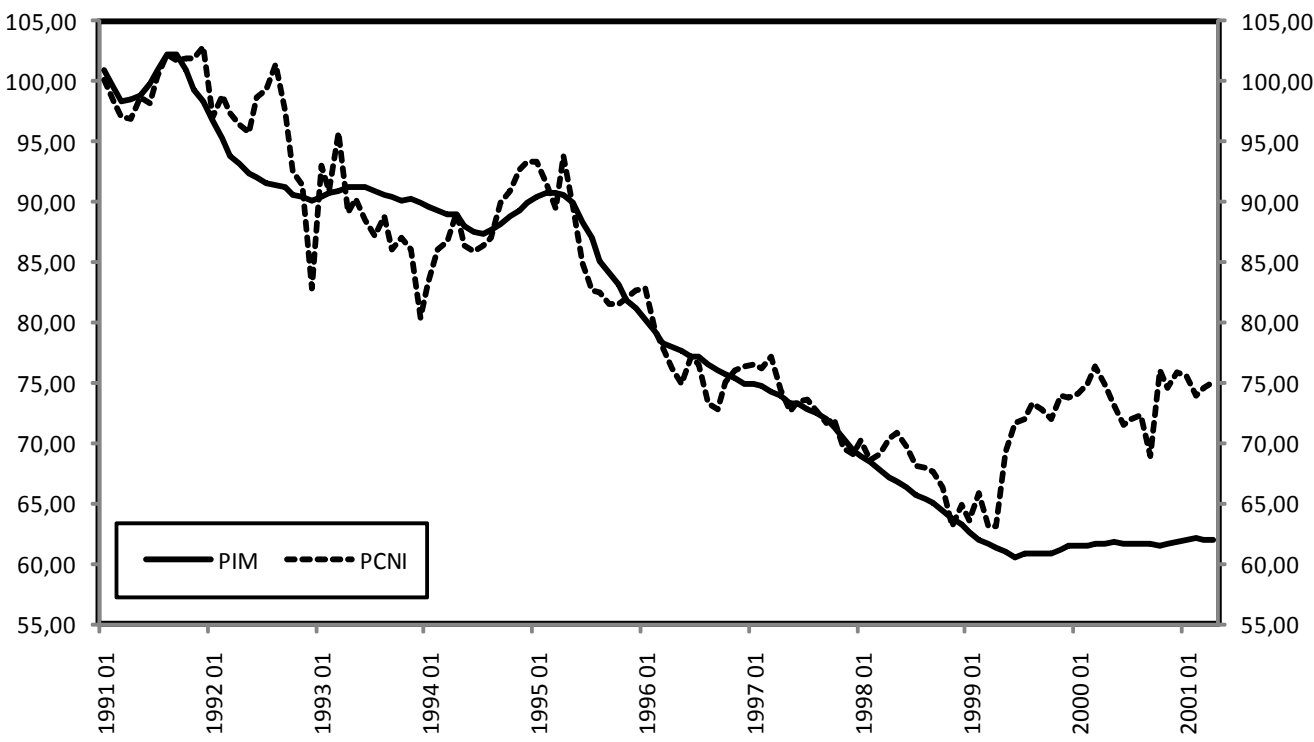

7 As taxas de crescimento do emprego, do custo médio do trabalho e das vendas reais foram mensuradas usando a taxa de variação na forma $g_{i t}=\left(x_{i t}-x_{i t-1}\right) /\left[\left(x_{i t}-x_{i t-1}\right) / 2\right]$, cujo resultado ficou limitado ao intervalo -1 a 1 . Se $g_{i t}>0$ houve crescimento do emprego na unidade produtiva, ao passo que $g_{i t}<0$ indica redução no emprego. 
Esse resultado sinaliza que uma proporção significativa das empresas não altera o emprego de um trimestre para outro. Caso o façam, esse ajuste é pequeno e próximo de zero. Observa-se ainda, à direita do zero, que as taxas de crescimento do emprego apresentam um decaimento mais rápido se comparado àquele apresentado no lado esquerdo da origem.

De acordo com a teoria da demanda por trabalho (e.g. HAMERMESH, 1993), seja pela resolução de um problema de minimização de custos, seja pela maximização de lucros e sob diferentes tipos de expectativas, pode-se especificar uma função (potencialmente implícita) de demanda por trabalho $\left(n_{i t}\right)$ nas empresas $i$ no período $t$ na forma $F\left(\Delta n_{i t}, \Delta z_{i t}, \theta\right)=0$, onde $z_{i t}$ representa um vetor de choques de rentabilidade na empresa, e $\theta$ um vetor de parâmetros associados à tecnologia, custos de ajustamento e até demanda por produto. O vetor de choques de rentabilidade em geral é associado a choques de custos do trabalho $\left(\mathfrak{w}_{i t}\right)$, a choques de demanda ou produção $\left(y_{i t}\right)$ e a choques não observados $\left(\varepsilon_{i t}\right)$.

De acordo com Varejão e Portugal (2007), Hamermesh e Pfann (1996a), sob as hipóteses usuais de tecnologia, associadas a funções de produção contínuas e diferenciáveis, estruturas de custos de ajustamento convexas levam a uma associação contínua e suave entre os choques e a variação do emprego. Por outro lado, se a estrutura de custos de ajustamento for não-convexa, a função de demanda por trabalho implícita será descontínua, gerando uma distribuição de variação no emprego bastante diferenciada da de choques de demanda, custos de trabalho e choques não observados.

Passamos, dessa forma, a avaliar as distribuições de choques de rentabilidade observados, ou seja, as mudanças no valor da produção (medido pelas vendas reais, na Figura 3) e as mudanças no custo do trabalho (Figura 4). As distribuições das taxas de crescimento das vendas reais e do custo médio do trabalho são apresentadas nas Figuras 3 e 4, respectivamente. Observa-se que as distribuições possuem um formato de sino padrão, sugerindo uma suavidade nas taxas de crescimento e a existência de uma continuidade. São indícios de que não existe uma forte concentração em zero, como foi observado na distribuição de variações no emprego. 


\section{FIGURA 2 - TAXA DE CRESCIMENTO DO EMPREGO}




FIGURA 3 - TAXA DE CRESCIMENTO DAS VENDAS REAIS

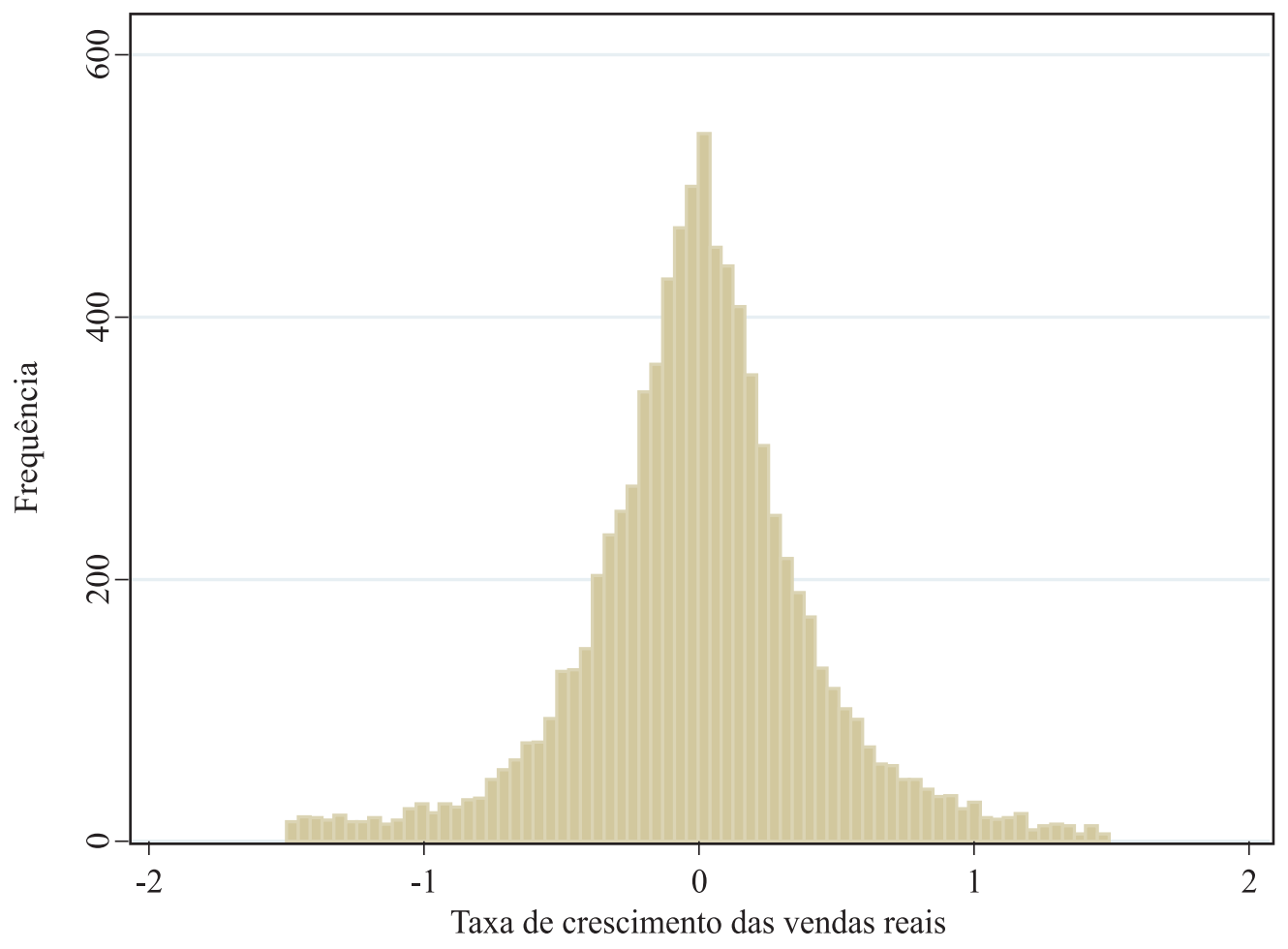

Para a distribuição de choques não observados o razoável é supor que seja contínua e suave, como a Normal ou até a Uniforme, como em Varejão e Portugal (2007). Seguindo estes autores, se as distribuições de choques forem suaves e contínuas, a existência de descontinuidades e concentração em zero é associada a estruturas de custos de ajustamento não-convexas. Assim, a metodologia empírica consiste em testar as possibilidades de concentração em zero de modo condicional. 


\section{FIGURA 4 - TAXA DE CRESCIMENTO DO CUSTO MÉDIO DO TRABA- $\mathrm{LHO}$}

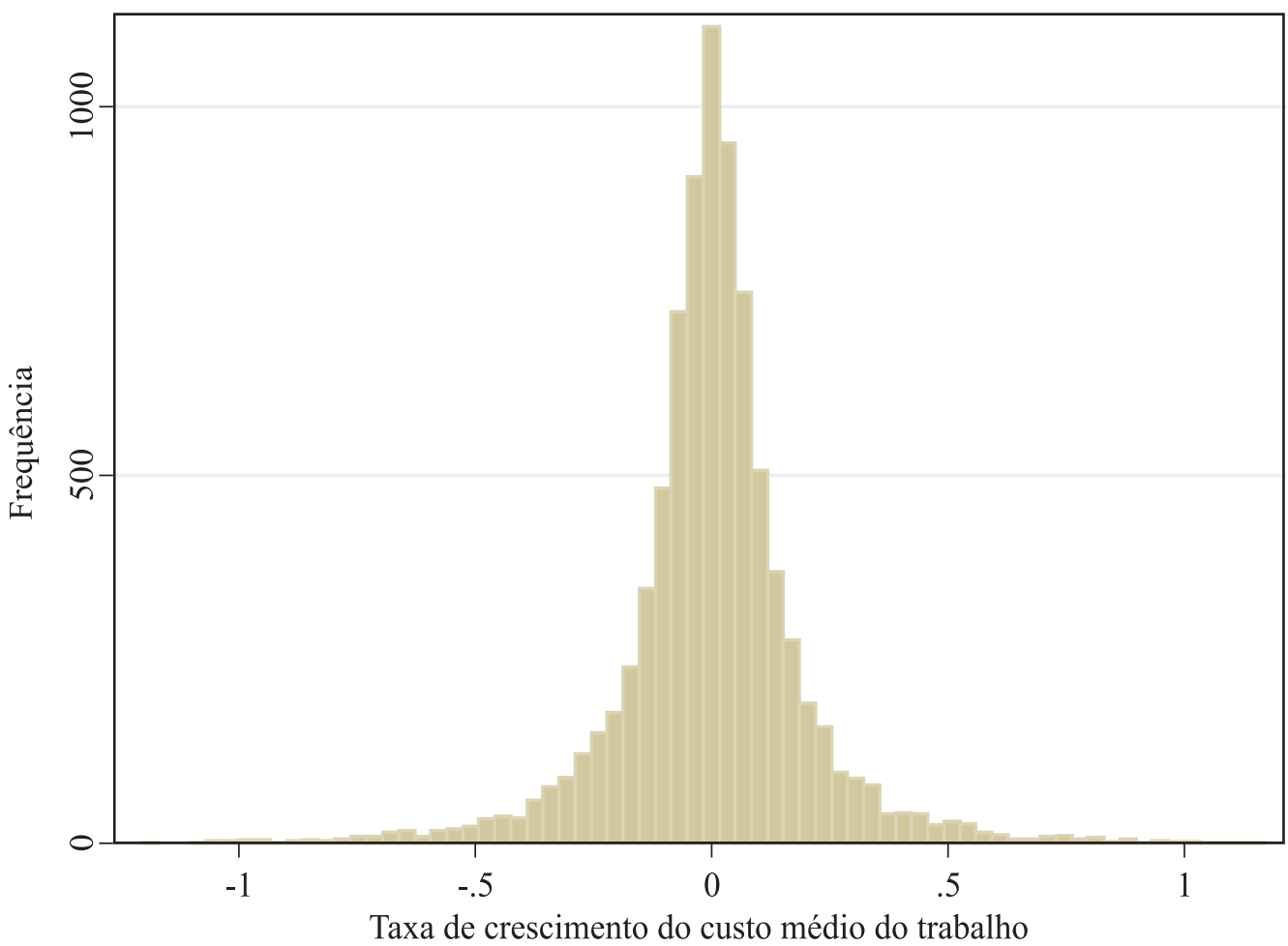

Duas metodologias complementares são empregadas para entender a dinâmica do ajustamento condicional aos choques correntes e passados. A primeira, uma matriz de transição, mede a probabilidade de entrada e saída da inatividade condicional à empresa estar ajustando ou não o emprego. A existência de custos de ajustamento convexos levaria a distribuições condicionais com baixa probabilidade de entrada e manutenção na inatividade. A segunda metodologia usa modelos de sobrevivência. A matriz de Transição de Markov impõe a hipótese de independência entre probabilidade de saída e duração no estado $e$. É uma hipótese muito forte e, além disso, incompatível com custos de ajustamento não-convexos. Sob este tipo de custos de ajustamentos, como por exemplo, custos fixos, os choques têm de acumular até o ponto de superar um valor limite e induzir a empresa a fazer um ajuste no seu número de funcionários. Assim, sob custos de ajustamento não-convexos haverá uma dependência da probabilidade de saída da inatividade (bazard, no sentido de probabilidade de saída da inatividade) da duração da inatividade (VAREJÃO; PORTUGAL, 2007). Um procedimento alternativo para definir o tipo de custo de ajustamento vem do teste do formato da função hazard. 


\section{MATRIZ DE TRANSIÇÃO ENTRE OS REGLMES DE EMPREGO}

Apesar da distribuição das taxas de crescimento do emprego apresentadas na Figura 2 sugerir que o padrão de ajustamento é não-convexo, faz-se necessário encontrar outras formas que venham corroborar ou não com essa evidência. A principal diferença observada entre os dois possíveis padróes de ajustamentos pode ser materializada com base na continuidade ou não do ajustamento. Uma maneira simples de identificar se os custos de ajustamento são convexos (VAREJÃO; PORTUGAL, 2007) é verificar se, após um período em que ocorreu um ajuste na demanda por trabalho, subsequentes períodos com ajustes são observados, tendo em vista que a intensidade desses ajustes deve ser decrescente ao longo do tempo (isto é, $\delta n_{t+1} \neq$ 0 , dado que $\delta n_{t} \neq 0$ e que $\left.\delta n_{t+1}<\delta n_{t}\right)$. De maneira similar, se os custos de ajustamentos são não-convexos, os períodos subsequentes a um período de ajuste no emprego serão caracterizados por uma inatividade, ${ }^{8}$ ou seja, a empresa não irá promover nenhuma alteração no emprego (isto é, $\delta n_{t+1}=0$, dado que $\delta n_{t} \neq 0$ ). Portanto, observar se existiu ou não continuidade no ajustamento do emprego nos períodos subsequentes ao de um período de ajustamento pode ser um critério preliminar útil e rápido para identificar o padrão de ajustamento e, consequentemente, o tipo de custos de ajustamento no nível das firmas.

Assim, para distinguir se a estrutura de custos de ajustamentos é convexa ou nãoconvexa, todas as firmas em cada trimestre foram classificadas dentro de um regime de emprego e a sua situação a um período à frente foi observada. Foram considerados três regimes: $i)$ um regime de ajuste para cima $\left(\delta n_{t}>0\right)$; ii) um regime de ajuste para baixo $\left(\delta n_{t}<0\right)$ e, por fim, caso a firma opte por manter o mesmo nível de emprego, o que equivale a não realizar o ajustamento, um regime de inatividade $(I$, ou $\left.\delta n_{t}=0\right)$. Essas informações podem ser analisadas de acordo com as probabilidades que governam as transiçóes entre os regimes a partir de dados sobre as firmas que se movimentaram de um regime observado num determinado trimestre para outro no trimestre subsequente. Com essas informações é possível estimar as probabilidades condicionais, como por exemplo:

$$
P\left(I_{t} \mid \delta n_{t-1}>0\right)=\#\left(\delta n_{t-1}>0, \delta n_{t}=0\right) / \#\left(\delta n_{t-1}>0\right)
$$

onde $\#\left(\delta n_{t-1}>0, \delta n_{t}=0\right)$ é o número de empresas que ajustaram para cima o emprego em t-l e ficaram inativas no período $t$; e $\#\left(\delta n_{t-1}>0\right)$ indica o número de empresas que ajustaram o emprego para cima no período $t$ - 1 , isto é, a probabilidade amostral de não ocorrerem alterações no emprego em $t$, dado que as firmas ajustaram o emprego para cima em $t-1$.

8 Inatividade pode ser definida como não havendo alterações no nível de emprego da firma. 
Essas probabilidades são conhecidas como probabilidades de transição. Considerando $i, j=\left(\delta n_{t-1}>0\right),\left(\delta n_{t}<0\right)$ e $I$, elas mostram as probabilidades de transitar de um regime $j$ para um regime $i$ entre trimestres e são relativas às empresas, podendo ser estimadas sem qualquer suposição adicional. ${ }^{9}$ Para obtê-las, inicialmente contabilizase o número de empresas que se movimentam de um regime para o outro a cada trimestre, gerando nove fluxos possíveis, como pode ser visto na Tabela 1. A partir daí, as probabilidades ${ }^{10}$ devem ser calculadas para cada trimestre pela relação entre o número de empresas em cada transição e o número total de empresas em cada regime original.

Após calcular as probabilidades de transição entre os regimes e construir uma matriz, a análise dos resultados recai sobre as probabilidades descritas na diagonal principal, na qual são observadas as probabilidades de transição dentro do mesmo regime. Com exceção da situação da transição de inativo-inativo (de $I$ para $I$ ), todas as outras situações na diagonal podem ser consideradas evidências favoráveis a uma estrutura de custos de ajustamentos convexa. Os indícios de custos de ajustamentos fixos, ou de uma estrutura de custos de ajustamentos não-convexos, podem ser observados na importância da coluna $I$, na qual são descritas as probabilidades de transitar de $(\delta n<0)$ para $I$ (alta probabilidade), de $(\delta n>0)$ para $I$ (alta probabilidade) e, estando no regime de inatividade, de se manterem nesse regime, ou seja, de $I$ para $I$ (alta probabilidade).

A Tabela 1 apresenta as probabilidades de transição entre os regimes. Inicialmente, deve-se considerar se há independência entre os regimes entre períodos. É uma hipótese claramente rejeitada por um teste Qui-quadrado de Pearson. Em outras palavras, as probabilidades de cada estado no período $t$ dependem de em que estado a empresa estava no trimestre anterior.

Observa-se pela diagonal principal que a probabilidade de as firmas se manterem dentro do mesmo regime está próxima de $50 \%$, exceto para a transição do regime de inatividade para inatividade (de $I$ para $I$ ), ou seja, $27,72 \%$ das firmas que visitaram o regime de inatividade num trimestre e ainda permaneceram nesse regime no trimestre seguinte. Embora este número seja menor que para os estados de ajuste para cima ou para baixo, deve ser lembrado que o regime de inatividade considera apenas um valor (o zero) dentro dos números Reais. Ao mesmo tempo, manter-se em inatividade implica que não há choques, se os custos de ajustamento forem convexos. Pelas Figuras 3 e 4, vemos que choques iguais a zero são pouco frequentes no total de observações. Desta forma, temos evidências de que os custos de ajustamento não são convexos.

9 Para a operacionalização das estimativas como matriz de transição markoviana, ao contrário, várias hipóteses são necessárias.

10 As probabilidades de transição apresentadas na Tabela 2 seguem $1 \geq p_{i j} \geq 0$ e $\Sigma_{j} p_{i j}=1$. 
Observa-se ainda, na coluna do regime de inatividade $(I)$, que um pequeno percentual de firmas que fizeram uma transição dos regimes de ajustamento para cima $\left(\delta n_{t-1}>0\right)$ ou para baixo $\left(\delta n_{t-1}<0\right)$ passaram pelo regime de inatividade, ou seja, a probabilidade de $\left(\delta n_{t-1}<0, I\right)=6,54 \%$ e $\left(\delta n_{t-1}>0, I\right)=5,99 \%$, respectivamente. Por outro lado, a baixa probabilidade de retorno à inatividade, uma vez feito o ajuste (bem menor que os resultados de VAREJÃO; PORTUGAL, 2007, por exemplo), sugerem que o grau de não-convexidade dos custos de ajustamento é relativamente fraco. Por fim, quanto à simetria, as probabilidades de saída de inatividade, seja para ajuste para cima, seja para baixo, são similares.

\section{TABELA 1 - MATRIZ DE TRANSIÇÃO PARA EMPREGO TOTAL (1991-2004) - INATIVIDADE $(\triangle N=0)$}

\begin{tabular}{llccc}
\hline \multirow{2}{*}{ Período } & \multicolumn{3}{c}{ Período $t$} \\
\cline { 3 - 5 } & & $\left(\delta n_{t}<0\right)$ & $I=\left(\delta n_{t-1}=0\right)$ & $\left(\delta n_{t}>0\right)$ \\
\hline \multirow{3}{*}{ Período } & $I=\left(\delta n_{t-1}<0\right)$ & 53.24 & 6.54 & 40.22 \\
$t-1$ & 37.63 & 27.72 & 34.65 \\
& $\left(\delta n_{t-1}>0\right)$ & 44.81 & 5.99 & 49.21 \\
\hline \multicolumn{2}{l}{ Qui-quadrado de Pearson $(4)=634.4944$} \\
\hline
\end{tabular}

Fonte: Baseado em amostra da pesquisa Fiergs/CNI e cálculos dos autores.

Pode-se argumentar que o limite de zero absoluto para caracterizar o regime de inatividade é rígido dada a fricção natural de trabalhadores (seja por aposentadoria, mortes, ou outras razões). Ao mesmo tempo, não se deve descartar a possibilidade de erros de medida. Muitas vezes, um ajustamento de $1 \%$ implica a diferença de um trabalhador (ou menos) de um trimestre para outro. Assim, experimentamos com um limite menos rígido para caracterizar o regime de inatividade, que é o limite de $5 \%$ da taxa de ajuste percentual do emprego. ${ }^{11}$ Naturalmente, um limite maior para o regime de inatividade irá aumentar a probabilidade dos regimes de inatividade nos períodos $t$ e $t-1$.

A Tabela 2 apresenta a matriz de transição calculada para este novo critério. Como pode ser visto pela diagonal principal da matriz para o limite de $5 \%$ no ajuste do emprego, $68,27 \%$ das firmas que visitaram o regime de inatividade num trimestre permaneceram nesse mesmo regime no trimestre subsequente. Esse resultado sugere uma persistência do regime de inatividade e mostra a relevância desse regime como principal destino para uma grande parte das firmas que realizam uma transição de um trimestre para o trimestre seguinte como consistente com a existência de custos

11 Experimentamos também com um limite de 10\%, mas os resultados não mudam qualitativamente. 
de ajustamentos fixos. A coluna I mostra que, das empresas que partiram dos regimes de ajustamento para cima $\left(\delta n_{t-1}>0\right)$ ou para baixo $\quad\left(\delta n_{t-1}<0\right)$, cerca de $50 \%$ visitaram o regime de inatividade no período seguinte. Este grande aumento de probabilidades, bastante superior ao aumento da probabilidade de inatividade, condicional à inatividade no período $t$ - 1 pode ser interpretado como justificativa para a necessidade de considerar a inatividade como dentro de um limite diferente de zero, dada a possibilidade de existência de erros de medida na variável de emprego.
TABELA 2 - MATRIZ DE TRANSIÇÃO PARA EMPREGO TOTAL (1991-2004) - INATIVIDADE $(|\Delta N|<5 \%)$

\begin{tabular}{lcccc}
\hline \multirow{2}{*}{ Período } & \multicolumn{3}{c}{ Período $t$} \\
\cline { 3 - 5 } & $\left(\delta n_{t-1}<-5 \%\right)$ & 29.71 & $I=(|\delta n|=5 \%)$ & $\left(\delta n_{t}>5 \%\right)$ \\
\hline \multirow{2}{*}{ Período } & $I=(|\delta n|=5 \%)$ & 16.31 & 48.41 & 21.88 \\
$t-1$ & $\left(\delta n_{t-1}>5 \%\right)$ & 25.83 & 68.27 & 15.42 \\
& \multicolumn{2}{l}{ Qui-quadrado de Pearson $(4)=447.9110$} & $\operatorname{Pr}=[0.0000]$ & 50.29 \\
\hline
\end{tabular}

Fonte: Baseado em amostra da pesquisa Fiergs/CNI e cálculos dos autores.

Em geral, os resultados mostram a importância e grande frequência do regime de inatividade, que seria associado a custos de ajustamento não-convexos. Mas como este método é bastante subjetivo na identificação dos custos, partimos na próxima seção para testes de hipóteses sobre os custos de ajustamento.

\section{MODELOS DE DURAÇÃO DO AJUSTAMENTO DO EMPREGO}

Antes de descrever o procedimento de estimação de uma função hazard, faz-se necessário apresentar como ela pode ser empregada para informar se a estrutura de custos de ajustamentos é convexa ou não-convexa, seguindo Varejão e Portugal (2007), que por sua vez seguem Cooper et al. (1999). O primeiro passo consiste em definir a variável de duração, que mostrará o tempo em que a firma irá permanecer no regime de inatividade. Observa-se cada firma individualmente e verifica-se o momento em que entra no regime de inatividade, acompanhando-a até o momento em que deixa esse regime. Assim, a variável duração corresponde ao número de períodos no qual a firma permaneceu após visitar o regime de inatividade.

Para estudar esse fenômeno da duração será empregada uma função bazard, que permite encontrar a probabilidade instantânea de ajustar o emprego em $t$, dado que o es- 
tabelecimento esteve inativo até $t$. Com base em Lancaster (1990), Varejão e Portugal (2007), e Addison e Portugal (1998) uma função hazard pode ser definida como:

$$
h(t)=\lim _{\Delta t \rightarrow 0} \frac{(P(t \leq T<t+\Delta t \mid T \geq t)}{\Delta t}=\frac{f(t)}{1-F(t)}=\frac{f(t)}{S(t)}
$$

em que $f(t)$ é a função densidade de probabilidade; $F(t)$ é a função de distribuição; $S(t)$ é chamada função de sobrevivência. Uma função importante é a função hazard integrada

$$
\Lambda(t)=\int_{0}^{t} h(u) d u
$$

que descreve a função de sobrevida simplesmente por

$$
S(t)=\exp \left(-\int_{0}^{t} h(u) d u\right)=\exp (-\Lambda(t))
$$

Como não existe um formato único para uma taxa hazard apropriado em todas as situações, vamos adotar o mesmo procedimento utilizado por Varejão e Portugal (2007), seguindo o caso mais popular que é o uso de uma distribuição Weibull (LANCASTER, 1990, CAMERON; TRIVEDI, 2005). Um modelo bazard Weibull convencional é dado por:

$$
h(t)=\rho \lambda^{\rho} t^{\rho-1}
$$

que implica a função de sobrevida $S(t)=\exp \left(-(\lambda t)^{\rho}\right)$ ) e uma função cumulativa hazard:

$$
\Lambda(t)=(\lambda t)^{\rho}
$$

A opção por uma função de distribuição Weibull deve-se à simplicidade com que permite testar diretamente a dependência da duração baseada apenas em seu formato por meio de um parâmetro, $\rho$. Por exemplo, uma dependência de duração negativa está condicionada a um $\rho<1$. Caso contrário, $\rho>1$ sugere taxas hazard crescentes no tempo. Contudo, se $\rho=1$, observa-se a existência de uma distribuição exponencial (ou seja, uma função bazard constante), em que a duração na inatividade não altera a probabilidade de saída (bazard) da inatividade.

Após um período de inatividade a firma pode sair desse regime expandindo o emprego ou reduzindo o seu nível de emprego. Para considerar esses dois tipos de saída, a função hazard pode ser definida para um destino específico denotado por 
$R$ : saída com aumento do emprego e saída com redução do emprego. A equação a seguir mostra como isso pode ser feito:

$$
h(t)_{r}=\lim _{\Delta t \rightarrow 0} \frac{(P(t \leq T<t+\Delta t, R=r \mid T \geq t)}{\Delta t}
$$

a qual resulta numa função hazard agregada:

$$
h(t)=\sum_{j=1}^{2} h_{j}(t)
$$

e na seguinte função de sobrevida

$$
S(t)=\prod_{j=1}^{2} S_{j}(t)
$$

em que $S_{j}(t)=\exp \left(-\Lambda_{j}(t)\right)$ e $\Lambda_{j}(t)=\int_{0}^{t} h_{j}(u) d u$.

Na implementação empírica do modelo é levada em conta a definição do regime de inatividade apresentada na seção anterior: limite zero ou ajustamento do emprego líquido zero e limite de $5 \%$ sobre o ajustamento do emprego líquido. ${ }^{12}$ Evidências favoráveis a uma estrutura de custos de ajustamentos convexa estão condicionadas a um parâmetro $\rho=1$, sugerindo uma taxa hazard constante.

Intuitivamente, após receber um choque nas condições de demanda, a empresa inicia um processo de ajuste do emprego que ocorre lentamente e minimiza movimentos bruscos, que geram grandes custos de ajustamentos. Os choques que porventura estão ocorrendo antes de a empresa terminar o processo de ajuste fazem com que esse processo seja contínuo sem interrupções e que os ajustes não sejam dependentes do tempo.

Por outro lado, se os custos não forem convexos, ante uma distribuição uniforme no tempo de choques de demanda, o ajustamento não será independente no tempo, pois terá de haver um acúmulo de choques para que seja induzido um ajuste. Desta forma, quanto maior o tempo de inatividade, maior o acúmulo de choques e maior a chance de ajustamento. Assim, ao invés de $\rho=1$, temos $\rho<1$ ou $\rho>I$, sendo as taxas hazard decrescentes ou crescentes no tempo, ou seja, dependentes da duração, sugerindo que o padrão de ajustamentos seja não-convexo.

12 Como na seção anterior, foi experimentado o limite de $10 \%$ para o regime de inatividade sem mudanças qualitativas fora do esperado. 
Os resultados do ajuste de uma função hazard, considerando as duas definições alternativas para os regimes de não-ajustamento, são descritos a seguir. Os resultados são apresentados para períodos de cinco anos (rolling window), tendo em vista verificar se ocorreu alguma alteração em termos de não-ajustamentos em alguma das janelas, ${ }^{13}$ por mudanças tecnológicas ou institucionais. Por fim, os resultados foram tabulados de forma que numa tabela apenas as saídas da inatividade com expansão do emprego fossem consideradas, ao passo que, em outra, fossem considerados os resultados da saída da inatividade com queda no emprego. Esse procedimento permite obter informações sobre a existência ou não de assimetrias na saída da inatividade.

As Tabelas 3 e 4 apresentam os resultados do modelo de duração, observando a saída da inatividade com expansão no emprego e a saída da inatividade com uma queda no emprego, respectivamente. Como pode ser visto, de modo geral, independentemente de qual modo de saída seja observado, os valores estimados do parâmetro $\lambda$ da distribuição Weibull são inferiores a 0.5 , indicando uma baixa probabilidade condicional de abandonar o regime de não-ajustamento em qualquer uma das janelas ao longo de todo o período.

Outra informação importante pode ser obtida ao serem comparadas as probabilidades condicionais entre os dois modos de saída da inatividade. Observa-se que não existe um comportamento padrão entre as probabilidades de saída da inatividade com expansão daquelas de saída com queda no emprego para o critério de não-ajustamento definido como limite de $5 \%$. Apenas para o critério limite de zero absoluto as janelas com início até 1997 apresentam uma probabilidade maior para a saída com expansão do emprego, ao passo que para as janelas de 1997-2001, 1998-2002 e 1999-2004 ocorre o contrário, ou seja, as probabilidades de saída com expansão mostraram-se inferiores àquelas obtidas para saída com queda no emprego. Em termos percentuais, as diferenças entre as magnitudes dos coeficientes estimados, considerando apenas o limite de zero absoluto, variam de $59 \%$ a $137 \%$.

De qualquer maneira, esse resultado é interessante e permite inferir sobre a existência de indícios de que o tempo do ajustamento do emprego não apresenta um caráter simétrico, ou seja, as probabilidades da saída da inatividade com expansão do emprego são diferentes da saída com a queda de emprego. Buscando validar essa suposição, foi realizado o teste da razão da verossimilhança, no qual foi admitida a hipótese nula da inexistência de assimetria. Como pode ser observado na Tabela 4 , para um nível de $5 \%$ de significância, a hipótese nula foi rejeitada, o que permite inferir pela existência de assimetria.

13 A intenção não é fazer uma análise temporal, porém, ao proceder dessa maneira é possível verificar se existe alguma diferença caso escolhêssemos analisar um período qualquer. Dito de outra forma, permite termos uma ideia sobre se houve ou não uma quebra estrutural. 


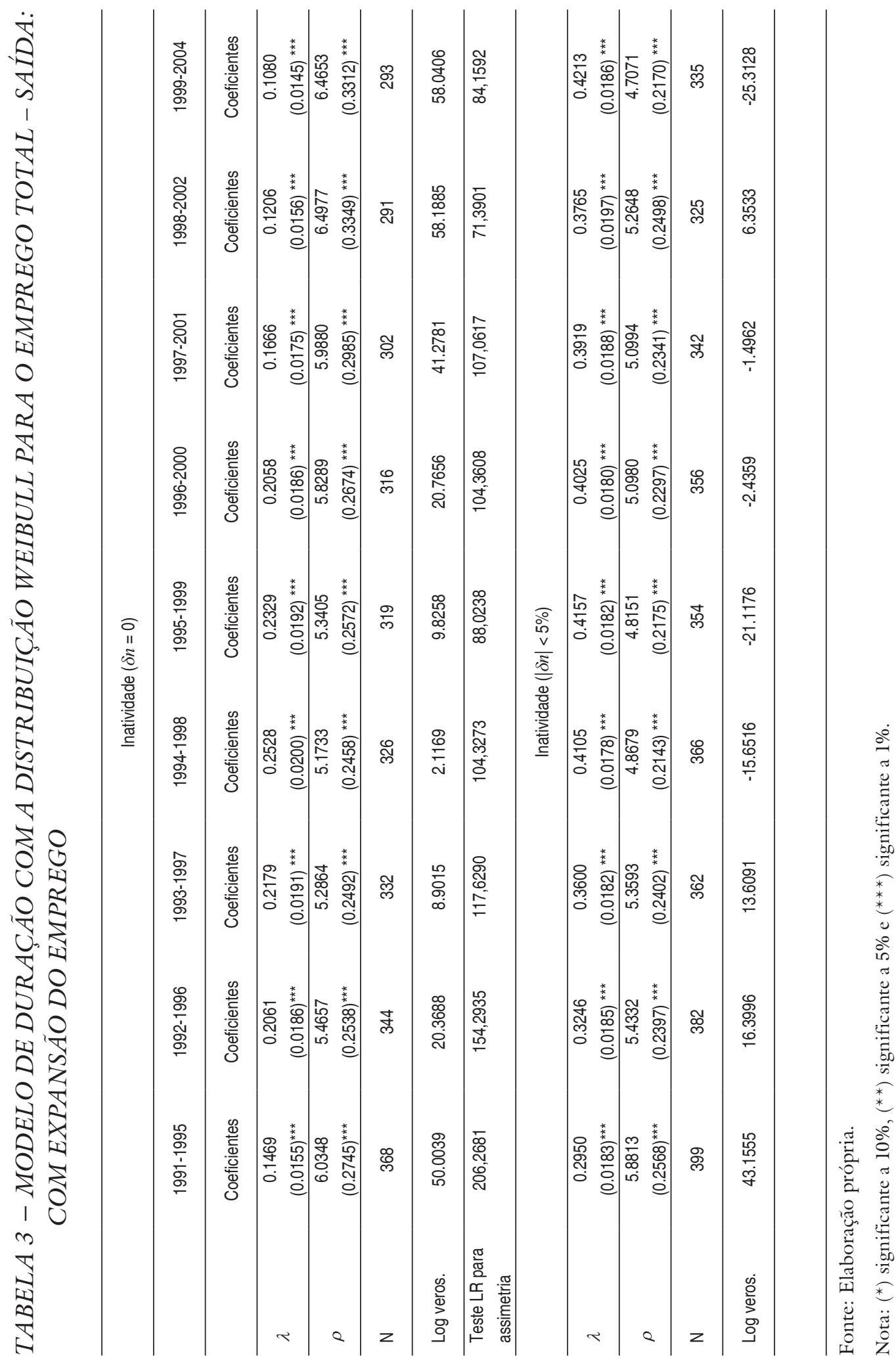




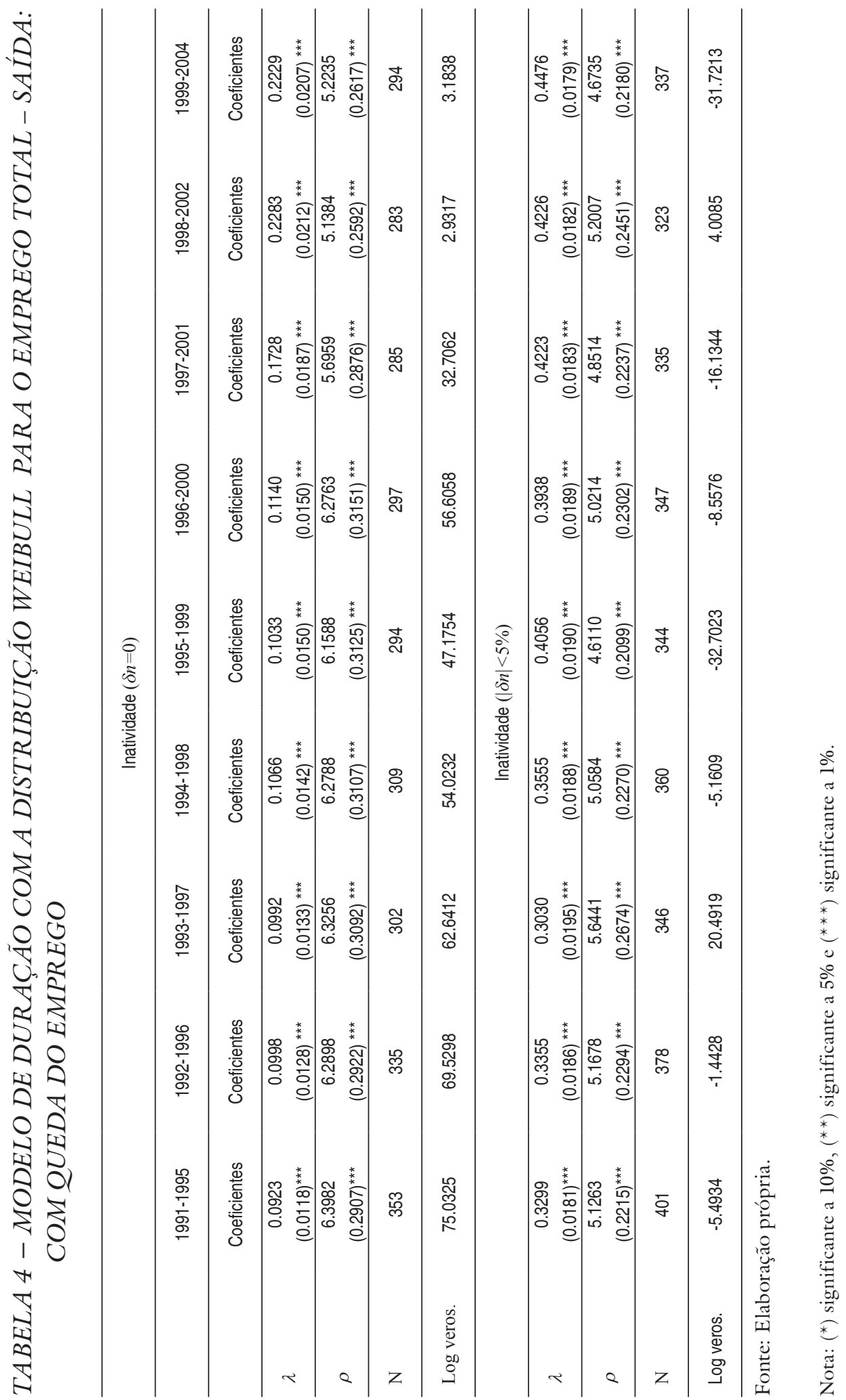


As probabilidades condicionais entre os dois critérios de inatividade, o limiar de zero absoluto e $5 \%$ adotados, também podem ser comparadas. Nesse caso, observa-se que as probabilidades para o limite de $5 \%$, em relação ao de zero absoluto, apresentam uma magnitude maior, como esperado. Esse padrão se mantém ao serem comparadas as probabilidades entre os dois modos de saída da inatividade, seja para expansão, seja para redução no emprego. De qualquer forma, à medida que aumenta o threshold, a probabilidade condicional de sair da inatividade também aumenta.

A priori os resultados acima sugerem que o padrão de ajustamento é não-convexo, como pode ser observado pelo parâmetro $\rho$ estimado. Observa-se que a função hazard Weibull, Tabelas 3 e 4, possui uma duração com dependência positiva, seja para as saídas da inatividade com expansão ou queda no emprego, seja para os threshold de zero absoluto ou de $5 \%$. Como o $\rho$ estimado é sempre maior do que um, a hipótese de uma hazard constante, $\rho=1$, é fortemente rejeitada, vindo a corroborar a hipótese de não-convexidade na função de custos de ajustamento. Intuitivamente, isso significa que um período de ajustamento deve ser seguido por um período de inatividade. Dessa forma, os ajustamentos feitos pelas firmas caracterizam-se por uma curta extensão de tempo e, quando ocorrem, são feitos através de alterações significativas do emprego, sugerindo períodos de nenhum ajustamento.

\section{CONSIDERAÇÕES FINAIS}

Diferentemente dos estudos encontrados na literatura brasileira, nos quais modelos de demanda por trabalho são estimados partindo de uma suposição da estrutura de custos de ajustamento do emprego (convexo ou não-convexo) não testada, este artigo buscou responder qual é a estrutura de custos de ajustamentos em nível das firmas na indústria de transformação, usando dados de empresas do Rio Grande do Sul.

Inicialmente, a descrição das taxas de crescimento do emprego para o pessoal total empregado deu indícios de um possível não-ajustamento ao apresentar uma grande concentração de taxas iguais a zero, ou seja, sugerindo a manutenção do nível de emprego. Esta grande concentração não se verificou nos dados associados a choques em uma função de demanda por trabalho (choques de custo do emprego e produção).

O artigo seguiu uma metodologia empírica proposta por Varejão e Portugal (2007), que faz a associação entre a probabilidade condicional de se manter em inatividade e uma possível dependência temporal da probabilidade de sair da inatividade com testes para identificar custos de ajustamento não convexos no emprego industrial. A ferramenta empírica é a estimação de funções hazard para saída da inatividade. O procedimento foi estimar uma função hazard, considerando duas possibilidades de 
saída da inatividade, a saber: saída com expansão do emprego e saída com redução do emprego.

Os resultados mostraram evidências favoráveis à função de custos de ajustamentos não-convexa, reforçando as evidências apresentadas pela matriz de transição para o limite de 5\%, pois identificou-se uma probabilidade maior de saída da inatividade quanto maior o período de duração neste regime. Supondo uma distribuição suave no tempo de choques, esta probabilidade crescente de saída quanto maior o tempo na inatividade está associada ao acúmulo de choques necessário para que seja economicamente ótimo o ajuste no emprego, em relação a custos de ajustamento fixos.

Em suma, as evidências empíricas apresentadas sugerem fortemente que a estrutura de custos de ajustamentos ao nível das firmas da indústria de transformação do Rio Grande do Sul, para o período analisado, é não-convexa. Embora para as análises da demanda por emprego publicadas no Brasil que usam dados setoriais (agregados) os custos de ajustamento possam ser considerados quadráticos e/ou convexos, pela suavização das não-convexidades, estudos que foquem o comportamento do emprego industrial com dados de empresas devem considerar funções de custos de ajustamento não-convexas, como custos fixos.

Por fim, como indicação para futuros trabalhos pode-se considerar a expansão da análise para outras amostras, seja em termos de cobertura regional, seja nacional e com outro perfil de tamanho de empresas. Todavia, deve-se notar que resultados de não-convexidade dos custos de ajustamento foram encontrados na literatura internacional empregando bases de dados com diferentes perfis de tamanho de empresas (e outras metodologias), como Hamermesh e Pfann (1996a) Del Boca, Galeotti e Rota (2003), Rota (2004), Ejarque e Portugal(2007)

\section{REFERENCIAS}

ADDISON, J.; PORTUGAL, P. Some specification issues in unemployment duration analysis. Labour Economics, v. 5, p. 53-66, 1998.

AMBRÓZIO, A. M. H. P. Custos de ajustamento e demanda dinámica por trabalho. 1999. Dissertação (Mestrado) - Departamento de Economia, Pontifícia Universidade Católica, Rio de Janeiro, 1999.

CABALLERO, R. J.; ENGEL, E. M. R. A. Microeconomic adjustment hazards and aggregate dynamics. Quarterly Journal of Economics, v. 108, p. 359-383, 1993.

; HALTIWANGER, J. Aggregate employment dynamics: building from microeconomic evidence. The American Economic Review, v. 87, p. 115-137, Mar. 1997. 
CAMERON, A.C.; TRIVEDI, P. K. Microeconometrics: methods and applications. Cambridge University Press, 2005.

COOPER, R. HALTIWANGER, J.; POWER, L. Machine replacement and the business cycle: lumps and bumps. American Economic Review, v. 89 p. 921-946, 1999.

COOPER, R. W.; WILLIS, J. L. The economics of labor adjustment: mind the gap. NBER Working Paper, n. 8527, 2001.

CORSEUIL, C.H.; SERVO, L.M.S.. (Org.). Criação, destruiçãa e realocação de empregos no Brasil. Brasília: IPEA, 2007.

DAVIS, S. J.; HALTIWANGER, J. Gross job flows. In: ASHENFELTER, O.; CARD, D. (Ed.). Handbook of Labor Economics, v. 3, Netherlands: Elsevier Publishers, 1999.

DEL BOCA; GALEOTTI; ROTA. Non-convexities in the Adjustment of Different Capital Inputs: A Firm-level Investigation. Fondazione Eni Enrico Mattei, 2003. (Working Paper n. 35).

EJARQUE, J. M.; PORTUGAL, P. Labor adjustment costs in a panel of establishments: a structural approach. The Institute for the Study of Labor (IZA), 2007. (Discussion Paper, 3091).

ESTEVÃO, M. M. Emprego, jornada média e custo de ajustamento da mão-de-obra: um modelo teórico e estimativas para as indústrias brasileiras e paulista. Rio de Janeiro: IPEA, 1991. (Cadernos de Economia, n. 6).

GONZAGA, G.; CORSEUIL, C. H. Emprego industrial no Brasil: análise de curto e longo prazo. Revista Brasileira de Economia, v. 55, n. 4, p. 467-491, out./dez. 2001 .

HAMERMESH, D. S. Labor demand and the structure of adjustment costs. The American Economic Review, v. 79, p.674-689, Sept. 1989.

. Aggregate employment dynamics and lumpy adjustment costs. CarnegieRochester Conferences Series on Public Policy, v. 33, p. 93-130, 1990.

. A general model of dynamic labor demand. The review of Economics and Statistics, v. 74, n. 4, p. 733-737, 1992.

. Labor demand. Princeton University Press, 1993.

; PFANN, Gerard A. Adjustment costs in factor demand. Journal of Economic Literature, v. 34, p. 1264-1292, Sept. 1996a.

; PFANN, Gerard A. Turnover and the dynamics of labour demand. Economica, v. 63 , p. 359-367, 1996 b.

LANCASTER, T. The econometric analysis of transition data. Cambridge: The MIT Press, 1990. 
MAGNUSSON, L. Demanda por mão-de-obra no setor industrial brasileiro - 1949/95. 2000. Dissertação (Mestrado em Economia) - Departamento de Economia, Universidade de São Paulo, São Paulo, 2000.

MEYER, J. B. Custo de ajustamento e demanda por trabalho no Brasil: uma estimativa setorial. Dissertação (Mestrado em Economia) - Departamento de Economia, Pontifícia Universidade Católica, Rio de Janeiro, 1998.

NICKELL, S. J. Dynamic models of labour demand. In: ASHENFELTER, O; LAYARD, R. (Ed.). Handbook of labor Economics. v. 1, Netherlands: Elsevier Publishers, p. 473-522, 1986.

NIELSEN, O; SCHIANTARELLI, F. Zeroes and lumps in investment: empirical evidence on irreversibilities and non-convexities. Review of Economics and Statistics, v. 85, p. 1021-1037, 2003.

OI, W. Y. Labor as a quasi-fixed factor. Journal of Political Economy, v. 70, p. 538-555, 1962.

PEREIRA, R.; GONZAGA, G. Demanda dinâmica por emprego e horas: uma aplicação do modelo linear-quadrático. Rio de Janeiro: IPEA, DIPES, 1998. (Série Seminários IPEA, n. 17).

PEREIRA, R. M. Demanda dinâmica por emprego e horas e a questão da partilha do trabalho: aplicações do modelo linear-quadrático. 1998. Dissertação (Mestrado em Economia) - Departamento de Economia, Pontifícia Universidade Católica, Rio de Janeiro, 1998.

ROTA, P., Estimating labor demand with fixed costs. International Economic Review, v. 45, p. 25-48, 2004.

VAREJÃO, J.; PORTUGAL, P. Employment dynamics and structure of labor adjustment costs. Journal of Labor Economics, v. 25 n.1, p. 137-165, 2007. 







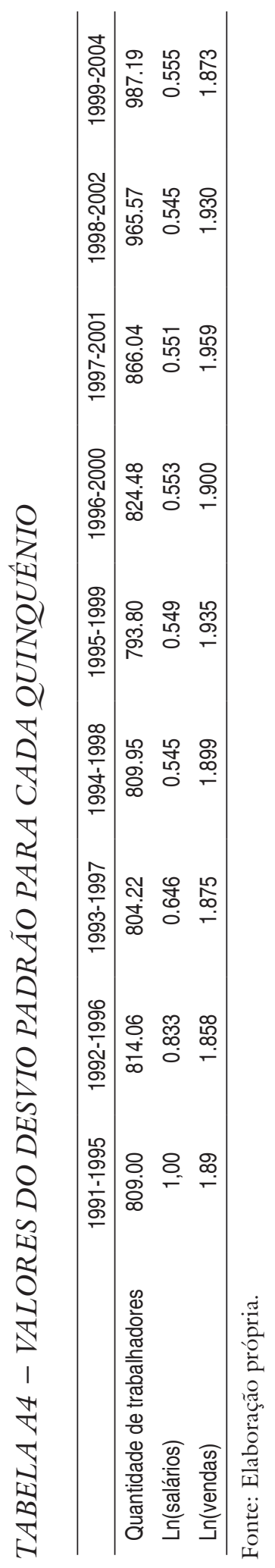

